\title{
Effectiveness of Repetitive Transcranial Magnetic Stimulation on Freezing of Gait in Parkinson's Disease Patients: A Systematic Review of the Literature
}

\author{
Wael Ibrahim, MD* \\ Department of Neurology, Kasr Alainy Hospital, Faculty of Medicine, Cairo University, Egypt
}

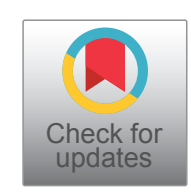

\begin{abstract}
Background: Episodes of FOG is often a debilitating event occurring in patients with Parkinson's disease (PD). TMS has been studies as a treatment options for a number of neuropsychiatric disorders including depression, PD, dystonia, and epilepsy.

Objectives: To assess systematically, the beneficial therapeutic effect of Transcranial magnetic stimulation (rTMS) on freezing of gait (FOG) in PD patients.

Methods: A systematic literature search of the PubMed, Scopus and Cochrane databases was performed. Randomized controlled trials, non-randomized controlled trials, retrospective studies and single arm studies of rTMS treatment on FOG in PD patients. A descriptive analysis of published studies was performed. The PubMed online search identified 7 publications, second search through Scopus identified 18 publications, and a third search through Cochrane identified 17 publications.

Results: A total of 17 Full-text articles assessed for eligibility with 9 articles excluded. Altogether, the included studies reported 96 patients with FOG. All trials implemented FOG Questionnaire being assessment tool for gait as primary and/ or secondary outcome, with few studies also used frequency of FOG episodes or TUG test to measure the improvement. A sham stimulation was used in five studies, all of them were cross over design except one study. The FoG-Q score showed significant improvement in $(n=3)$ studies, Meanwhile the UPDRS III and/or total score showed significant improvement in majority of studies $(n=5)$.

Conclusion: From the descriptive analyses, and from the available data of relatively small sample sized studies, in spite of the above limitations, rTMS had potential beneficial effect in improving the FOG in PD patients depending on the used protocol.
\end{abstract}

\section{Keywords}

Repetitive transcranial magnetic stimulation, Freezing of gait, Parkinson's disease, Parkinson plus syndromes, Neuromodulation

\section{Introduction}

Gait disorders in Parkinson's disease (PD) has several presentations including stooped posture, festination, shuffling steps, freezing of gait (FOG) and falls [1]. Episodes of FOG is often a debilitating event occurring mostly as a late feature in patients with PD [2], which usually disturbs their balance and results in subsequent falls [3]; and with prevalence within recent studies ranging from $21 \%$ to $38.2 \%$ [4]. FOG is defined as "a brief episode during which patients find it impossible to generate effective forward stepping movements, in the absence of a cause other than Parkinsonism or higher cortical deficits" [5]. Those events not only occur during turning and step initiation but also in situations where patients features physical barriers as a doorway or in stressful situations [2].
In terms of pathogenesis, FOG is still not clearly understood due to complex clinical presentation, and lack of dis-

*Corresponding author: Wael Ibrahim, MD, Department of Neurology, Kasr Alainy Hospital, Faculty of Medicine, Cairo University, 37 Elhassan street, Dokki, Giza, 12411, Egypt, Tel: +201111919927

Accepted: January 25, 2021

Published online: January 27, 2021

Citation: Ibrahim W (2021) Effectiveness of Repetitive Transcranial Magnetic Stimulation on Freezing of Gait in Parkinson's Disease Patients: A Systematic Review of the Literature. J Neurodegener Disord 4(1):101-112 
Citation: Ibrahim W (2021) Effectiveness of Repetitive Transcranial Magnetic Stimulation on Freezing of Gait in Parkinson's Disease Patients: A Systematic Review of the Literature. J Neurodegener Disord 4(1):101-112

tinctive pathological finding in postmortem specimens. However, FOG is not exclusive to PD and can be present in other disorders as in primary progressive freezing of gait and also more commonly encountered in other parkinsonian syndromes such as normal pressure hydrocephalus or vascular parkinsonism $[3,6]$. Meanwhile, responsiveness to dopaminergic medications in PD remains modest which may highlights the role of non-dopaminergic circuits in FOG pathogenesis in PD patients [7]. With the era of Multimodal neuroimaging; FOG seems to be related partially to interruption in the executive-attention network along with regional tissue loss including the premotor area, and inferior frontal gyrus [8].

There are three clinical patterns of FOG presentations: First, Trembling in place, second, shuffling forward, and third, total akinesia; all of them can be provoked by several situations which includes: 1) Freezing when turning and is referred as Turning hesitation; 2) Freezing when gait is initiated and is referred as Start hesitation; 3) Freezing when approaching target and is referred as Reaching destination hesitation; and 4) Freezing in open and narrow space and is referred as Open space hesitation and Tight quarter hesitation respectively $[3,6]$.

Due to the fact that FOG events are rarely encountered in clinics, a freezing of gait questionnaire (FOG-Q) had been implemented to increase accuracy of detecting the event rather than simply asking about them [3]. Other tools for gait assessment can be also implemented such as electromyography (EMG) and gait analysis lab using a three-dimensional (3D) camera [9]. In terms of medical treatments, it usually offers a limited benefit. People who are undertreated increasing the dopaminergic drugs dose is a reasonable option, while if FOG is during off state, several treatment regimens can be used. One regimen is additions of catechol-O-methyltransferase (COMT) inhibitor and/or monoamine oxidase (MAO) type-B inhibitor, others includes increase the frequency and/or dosage of levodopa, or performing deep brain stimulation (DBS) surgery which usually provides a reduction in off state [3].

Transcranial magnetic stimulation (TMS) is a non-invasive brain stimulation procedure that creates electrical energy across the scalp and skull via placing a small coil of wire on the scalp which produces a magnetic field that passes relatively painlessly through the tissues of the brain in order to modulates its activity $[10,11]$.

Research on TMS has been growing worldwide in the last few decades. TMS has been studies as a treatment options for a number of neuropsychiatric disorders including depression, Parkinson's disease (PD), dystonia (writer's cramp), and epilepsy [11,12]. Although, there is limited knowledge about the mechanisms of action of rTMS in PD [13], previous literature [14] reported the effectiveness of rTMS in treatment of PD motor symptoms. Different techniques and frequencies (both low and high) had been tried in PD, and although basal ganglia is a deep structure away from reach of stimulating coil, it still can be modulated via functional connections with cortical areas [13].

However, there is few contraindications to TMS use including cardiac pacemakers, implanted medication pumps, and Metallic foreign body in skull or brain; also, Children should not be enrolled as participants for rTMS studies without compelling clinical rationale [10]. In general, TMS is a safe procedure associated with few side effects including -but not limited to- headache, burning scalp sensation, and neck pains $[10,14]$.

\section{Methods}

We followed the Preferred Reporting Items for Systematic Reviews and Meta-Analyses (PRISMA) statement guidelines [15] during the preparation of this systematic review.

\section{Eligibility criteria}

\section{Inclusion criteria}

Trial or study type: Eligible articles included for this review: Randomized controlled trials (RCT), non-randomized controlled trials, retrospective studies, single arm studies, and case series of rTMS treatment on FOG in PD patients.

Intervention: Repetitive transcranial magnetic stimulation (rTMS) with all frequencies and latencies, with any number of sessions over any period of time, and applied to any site are eligible, either administered alone or with other antiparkinsonian therapy, and/or rehabilitation therapy. To narrow the heterogenicity among different TMS techniques; Continuous theta burst stimulation (cTBS), intermittent theta burst stimulation (iTBS), transcranial direct current stimulation (tDCS), or Electroconvulsive therapy (ECT) patients were excluded from this analysis.

Comparator: Placebo (sham control group) or no comparator group.

Population: Idiopathic PD (IPD), Vascular Parkinsonism (VP) or Parkinson plus syndromes (PPS) (progressive supranuclear palsy, multiple system atrophy, corticobasal degeneration, and also diffuse Lewy body disease) patients diagnosed with FOG, of any age group, and any gender is included.

Outcome: At least one of the following outcomes for FOG \pm motor functions: frequency of freezing episodes, Freezing of Gait Questionnaire (FOG-Q), FOG questionnaire (FOG Q) short form (SF) and Timed Up and Go test (TUG), and Movement Disorder Society Unified Parkinson's Disease Rating Scale motor score (MDS-UPDRS III) and/or total score (MDS-UPDRS total score).

Exclusion criteria: Non-English language studies, animal studies, studies whose full text article were not available, theses and conference papers, as well as ongoing trials were excluded.

Objective: To assess systematically, the beneficial therapeutic effect of rTMS treatment on FOG in PD and atypical Parkinsonism patients.

Study design: Systematic review.

\section{Information sources and search}

We searched for all published clinical trials using the following search terms "transcranial magnetic stimulation" AND "freezing of gait" AND "Parkinson's disease" in the following 
electronic databases: PubMed, SCOPUS, and Cochrane, from inception till December, 2018. Also, to learn more about the ongoing trials for rTMS in treatment of FOG in PD patients;

A separate search was done to learn more about the ongoing trials for rTMS in FOG; we searched clinicaltrials.gov "www.clinicaltrials.gov" (using the same previous search queries) for the following type of ongoing clinical trials: "Not yet recruiting, Recruiting, enrolling by invitation, Active \& not recruiting, completed (and unpublished)".

\section{Selection of studies}

The author will select eligible studies. Screening of search results will be performed in two steps:

- First, to screen title and abstracts against the selection criteria. Articles that were unclear from their title or abstract were reviewed against the selection criteria through the full text;

- Second, to retrieve and screen full-text articles of eligible abstracts for eligibility to systematic review.

\section{Data extraction}

Authors will extract the following data independently; First author, year of publication, study type, sample size, mean age, frequency of freezing episodes, characteristics of

\section{Flow chart of included and excluded articles in the systematic literature search}
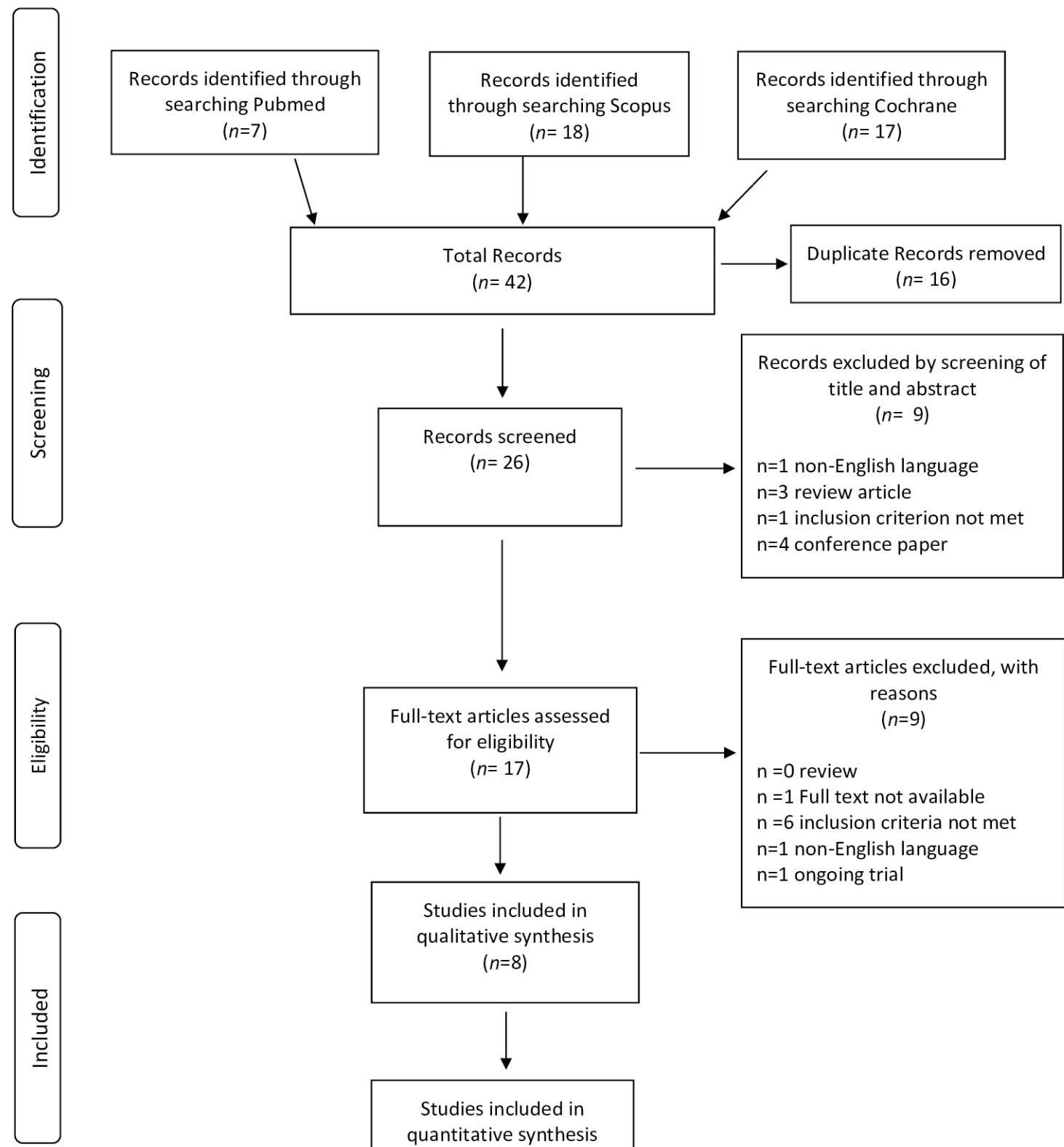

Studies included in

qualitative synthesis $(n=8)$

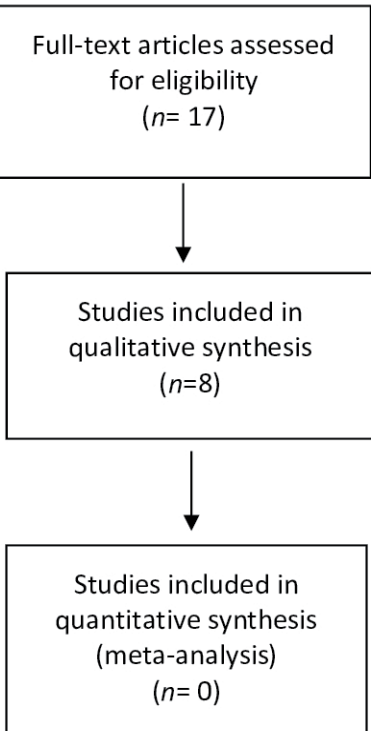

Full-text articles excluded, with reasons $(n=9)$

$\mathrm{n}=0$ review

$\mathrm{n}=1$ Full text not available

$\mathrm{n}=6$ inclusion criteria not met

$\mathrm{n}=1$ non-English language $n=1$ ongoing trial

Figure 1: Flow Chart of included and excluded articles in the systematic literature search. 
Citation: Ibrahim W (2021) Effectiveness of Repetitive Transcranial Magnetic Stimulation on Freezing of Gait in Parkinson's Disease Patients: A Systematic Review of the Literature. J Neurodegener Disord 4(1):101-112

rTMS sessions (number, frequency, intensity, and duration), and outcomes for FOG and motor functions using at least one of the following assessment tools: frequency of freezing episodes, FOG-Q, FOG-Q SF, MDS-UPDRS III and/or UPDRS total score, and TUG. Data will be exported from the online form as MS excel sheet.

\section{Statistical analysis}

We calculated the mean age and SD for all patients by using pooled mean and pooled SD equation according to Cochrane handbook of systematic reviews of interventions 5.1.0 (updated March 2011) [16]. When data are expressed as median and IQR, we used Hozo, et al. [17] at BMC Research Methodology equation to calculate or estimate mean and SD.

Data are expressed as means with SD (unless stated otherwise); Statistical results were considered to be significant when the $p$-value was less than 0.05. Data analysis was performed using Microsoft Office excel 2016 and Statistical Package for Social Science (SPSS) version 23 (IBM Corp., Armonk, NY, USA).

\section{Synthesis of data and analysis}

Due to heterogeneity and low sample size of studies, no statistically justified analyses - could be performed on the provided data. Hence, a descriptive analysis of the published studies was performed instead.

\section{Summary measures}

The search strings, list of relevant reviews, data coding, and the quality criteria used in this review can be requested from the corresponding author.

\section{Patient and public involvement}

Patients were not involved in setting the research question or the outcome measures, developing plans for design, or implementing the study. Also, no patients were asked to advice on interpretation or writing up of results. There are no plans to disseminate the results of this research to study participants or the relevant patient community.

\section{Results}

\section{Selection of articles}

The systematic literature search and subsequent selection are summarized in a flow diagram (Figure 1). The PubMed database online search identified 7 publications, a second database search through Scopus identified 18 publications, and a third database search through Cochrane identified 17 publications. After 16 duplicate publications were removed, a total of 26 publications were screened for title and abstract. All pre-clinical/animal studies, review articles, articles not written in the English language, as well as conference papers were excluded ( $n=9$ articles). A total of 17 full-text articles were assessed for eligibility, with 9 being excluded for the following reasons: Full text not available $(n=1)$, ongoing trial ( $n$ $=1)$, non-English language $(n=1)$, and inclusion criteria not met $(n=6)$.

\section{Study characteristics}

Characteristics of the included studies and patient outcome(s) are summarized in Table 1 and Table 2. A total of 8 studies from 2007 through 2018 were included in the descriptive analysis and systematic review. All trials were prospective studies. Among all trials, three studies were non-controlled/ single arm; and five were sham-controlled with crossover $(n=$ $4)$ and without crossover $(n=1)$.

\section{Baseline characteristics of included studies}

There were 96 participants in the total eight studies included in this systematic review. The number of patients per study ranged from 4 to 19 . Patients were mostly older age, with mean sample ages ranging from 63.70 to 74.57 years, and the pooled mean age for all participants in the total eight studies was 68.30 years.

Almost all studies recruited IPD patients except Chang and colleague [18] study, and Lee and colleague [19] study were both recruited Parkinson plus syndromes, with the former enrolled no IPD patients. From the total 96 participants included, IPD represented most of patients with $79.1 \%(n=76)$, while remaining patients either VP representing $11.4 \%$ ( $n=$ 11 ) or parkinsonian plus syndromes representing the minority of patients with $9.3 \%(n=9)$.

The rTMS current aim to target different regions among our review including motor cortex (MC), supplementary motor area (SMA), medial prefrontal cortex (MPFC) or dorsolateral prefrontal cortex (DLPFC). The leg and/or hand area are the targets within primary MC. The MC is the most common region of rTMS target present in 7 out of 8 studies; with less targeted regions -regarding number of studies- in descending manner is DLPFC $(n=2)$, SMA $(n=2)$, and $\operatorname{mPFC}(n=1)$. The frequency of rTMS used range from $1 \mathrm{~Hz}$ up to $25 \mathrm{~Hz}$ with most commonly used frequency used is $10 \mathrm{~Hz}$ (used in 5 studies). The intensity of stimulation used is reported in 6 studies, all of them used $90 \%$ intensity with exception of Thomassen and Arns [20] study used $120 \%$. The number of rTMS sessions given range from one session up to 16 sessions with calculated median value of 5 sessions; and usually given in frequency of 3-4 times per week, day after day or even daily sessions as in Rektorova and colleague [21] study.

A comparator or sham stimulation was used in five studies, all of them was cross over design except in El-tamawy and colleague [22] study were patient was randomized to either a real or sham stimulation group. Lee and colleagues [19] approached the patients differently in his study, were all patients had received real and sham stimulation in a cross over successive manner separated by interval period of 24 hour between each, with real stimulation to three different brain regions (total 4 sessions, one for each). The other three studies were non-controlled, both Kim S. J and colleague [23] study, and Rektorova and colleague [21] study assigned different patients for stimulation in different brain region for each group, with former stimulated MC or SMA, and latter stimulated MC or DLPFC region. Thomassen and Arns [20] study was the only single arm study to target single brain region (MC for both hand and leg area), and all over only three studies targeted a single brain region were $(n=2)$ studies are controlled. 


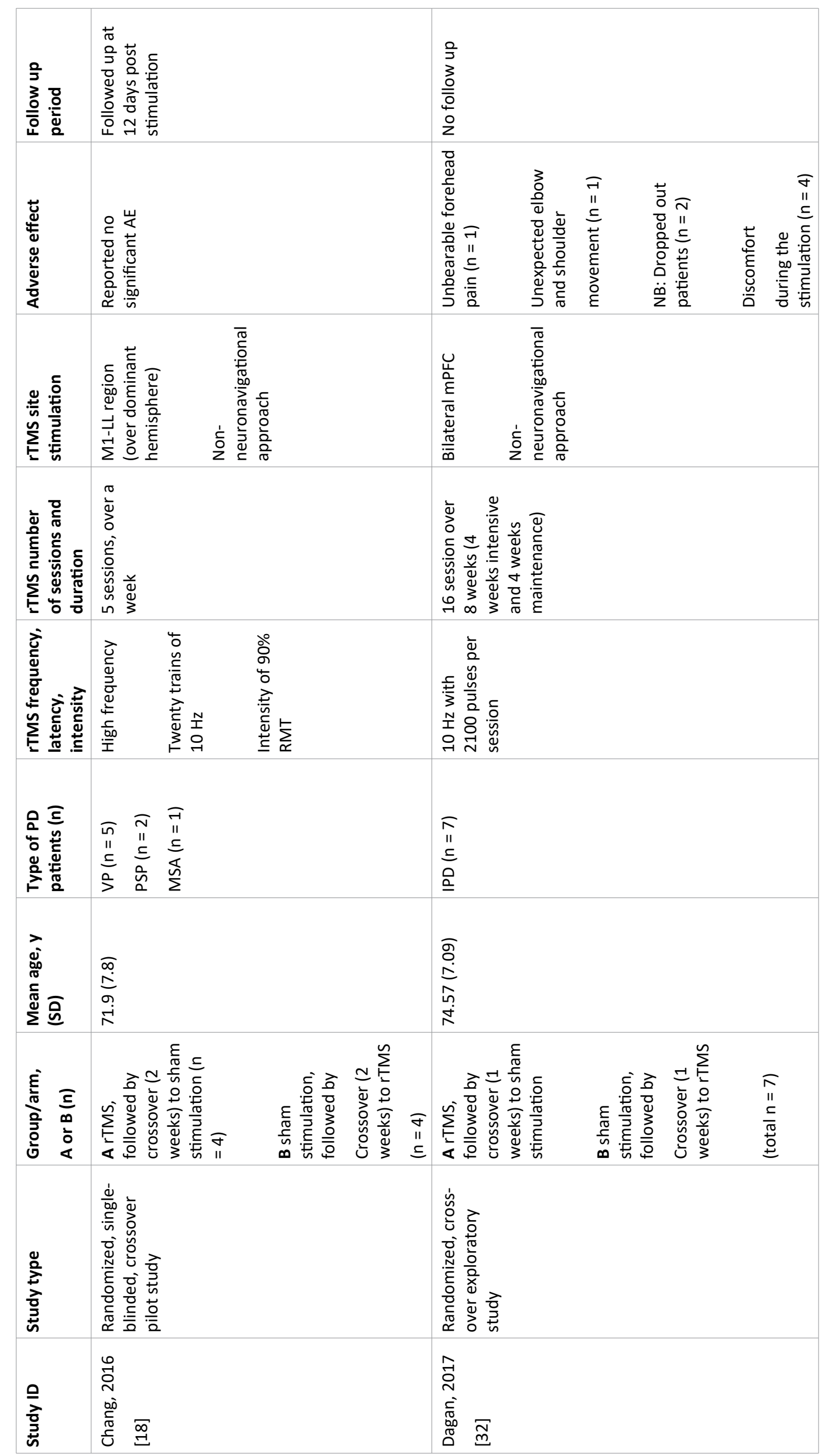


Citation: Ibrahim W (2021) Effectiveness of Repetitive Transcranial Magnetic Stimulation on Freezing of Gait in Parkinson's Disease Patients: A Systematic Review of the Literature. J Neurodegener Disord 4(1):101-112

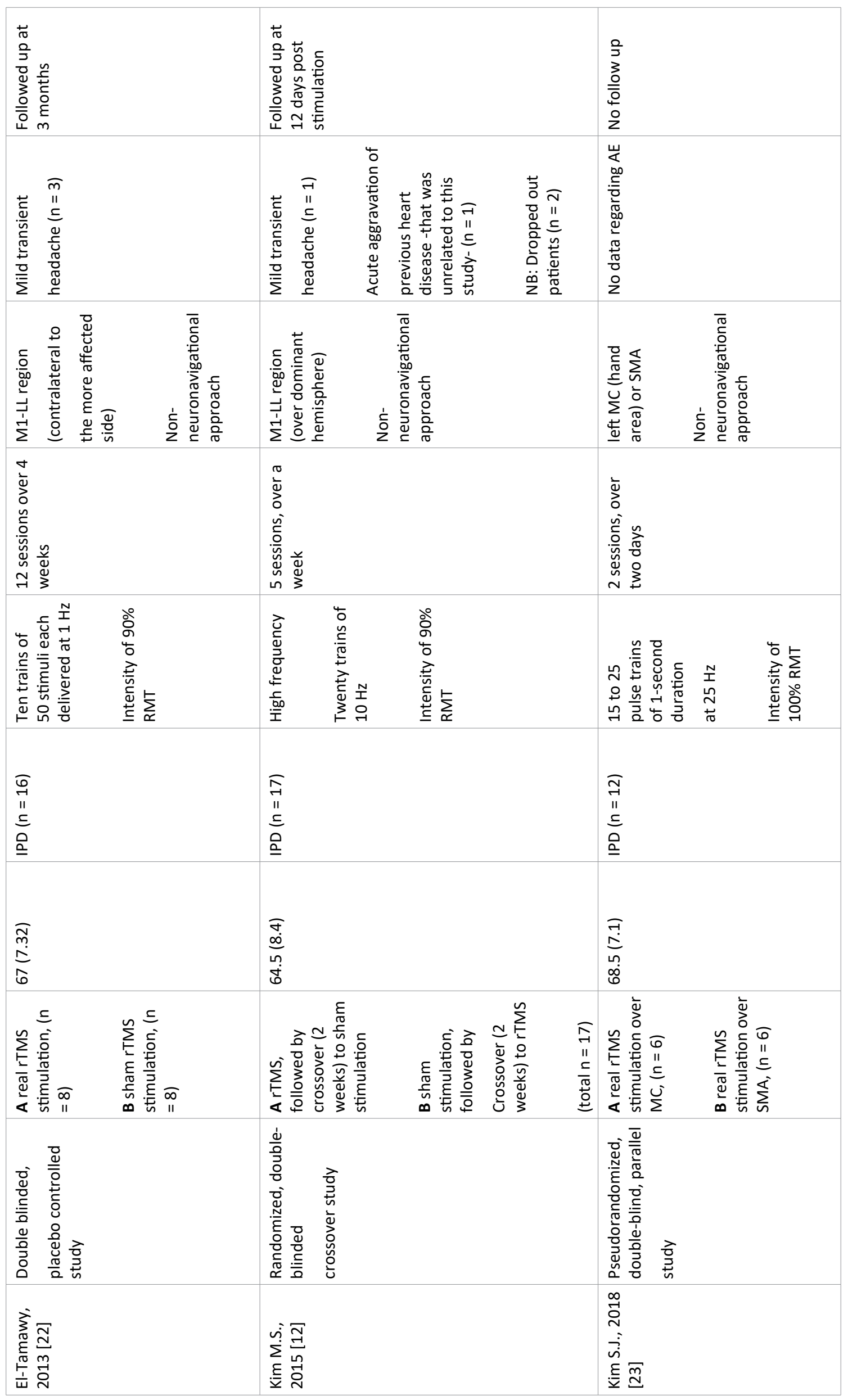




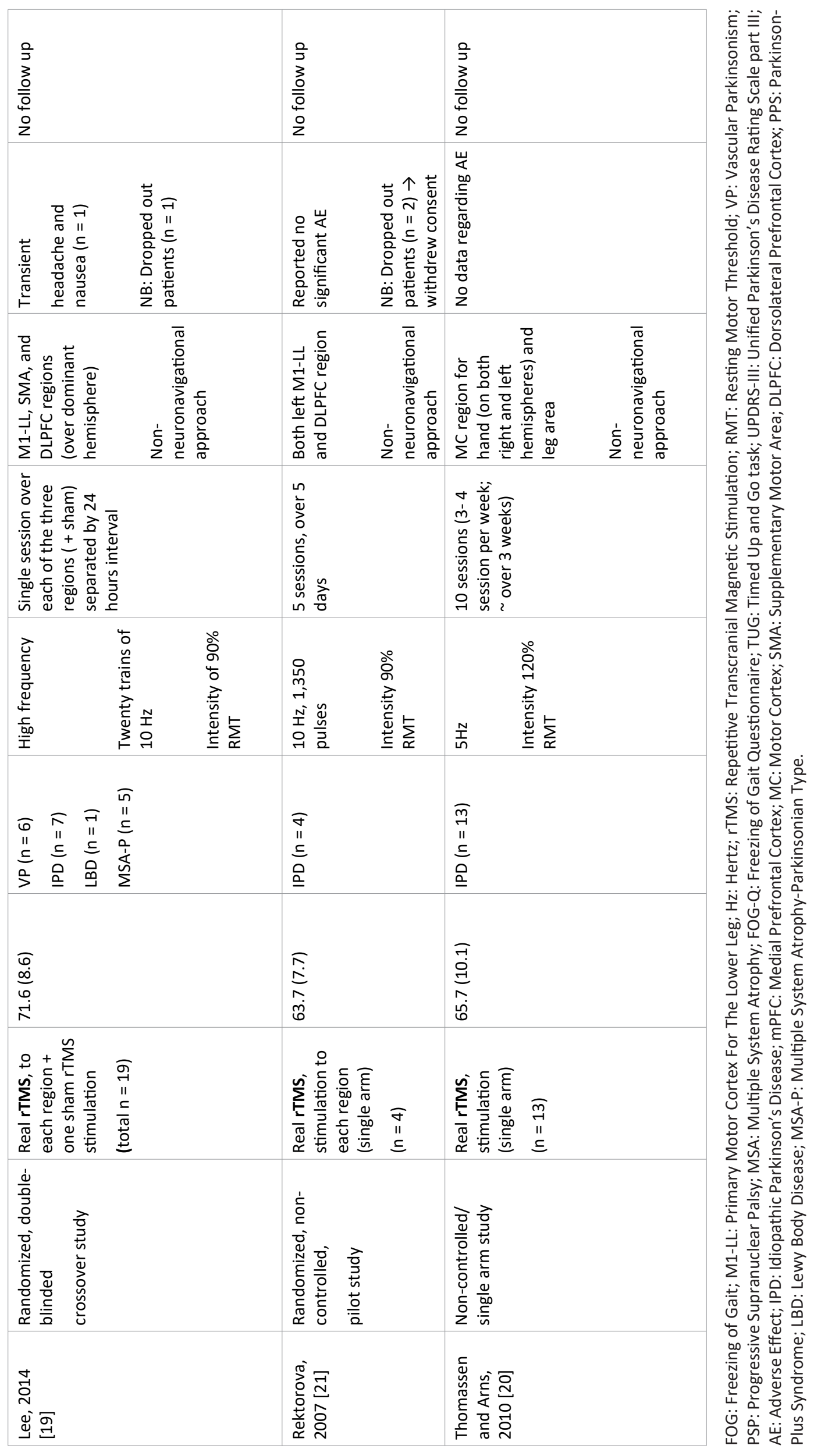


Citation: Ibrahim W (2021) Effectiveness of Repetitive Transcranial Magnetic Stimulation on Freezing of Gait in Parkinson's Disease Patients: A Systematic Review of the Literature. J Neurodegener Disord 4(1):101-112

Table 2: Outcome summary of included studies.

\begin{tabular}{|c|c|c|}
\hline Study ID & Primary Outcome(s) & Secondary Outcome(s) \\
\hline $\begin{array}{l}\text { Chang, } 2016 \\
{[18]}\end{array}$ & $\begin{array}{l}\text { Mean change in the FoG-Q score is by }-2.3 \text { points in real arm and }-0.4 \\
\text { in sham arm } \\
\text { Mean FoG-Q score in post-real rTMS arm } 13(4.2)(p=0.049) \\
\text { Mean FoG-Q score in post-sham rTMS arm } 15(4.5) \\
\text { Mean change in TUG task from } 53.75(31.88) \text { to } 46.12(24.60) \text { in real } \\
\text { rTMS arm }(p=0.049)\end{array}$ & $\begin{array}{l}\text { Improvement in mean UPDRS-III from } 20.1 \\
(12.0) \text { pre-stimulation to } 17.3(12.8) \text { post- } \\
\text { stimulation in real rTMS arm }(p=0.030)\end{array}$ \\
\hline Dagan, 2017 [32] & $\begin{array}{l}\text { Mean change in the FoG-Q score from } 19.67(1.86) \text { to } 18.67 \text { (3.27) in } \\
\text { real rTMS arm ( } p=0.197) \\
\text { Mean change in the FoG-Q score from } 19.86(3.13) \text { to } 21.29(3.15) \text { in } \\
\text { sham rTMS arm ( } p=0.063)\end{array}$ & $\begin{array}{l}\text { significant differences were observed in the } \\
\text { motor part of the UPDRS in real rTMS arm ( } p \\
=0.028) \text {, and not significantly affected by the } \\
\text { sham arm. }\end{array}$ \\
\hline $\begin{array}{l}\text { El-Tamawy, } 2013 \\
{[22]}\end{array}$ & $\begin{array}{l}\text { Mean Freezing episodes/day in real rTMS arm and sham rTMS arm was } \\
11.87(6.81) \text { and } 30(16.81) \text { respectively }(p=0.013) \\
\text { Mean FOG-Q in real rTMS arm and sham rTMS arm was } 10(3.42) \text { and } \\
20(4.63) \text { respectively }(p=0.001)\end{array}$ & $\begin{array}{l}\text { Total UPDRS score was not affected in either } \\
\text { study arms. } \\
\text { No serious adverse events were detected. } n= \\
3 \text { patients reported mild transient headaches } \\
\text { (one patient received real rTMS and the } \\
\text { other two were on the sham arm) }\end{array}$ \\
\hline $\begin{array}{l}\text { Kim M. S., } 2015 \\
{[12]}\end{array}$ & $\begin{array}{l}\text { change of FOG-Q at post-rTMS showed a significant improvement in } \\
\text { the real arm compared with the sham condition }(p<0.05)\end{array}$ & $\begin{array}{l}\text { At post-rTMS, the change of the UPDRS in the } \\
\text { real arm showed significant improvements } \\
\text { compared with the sham arm }(p<0.05) \\
\text { TUG and UPDRS-III at post-rTMS significantly } \\
\text { improved compared with a pre-rTMS in real } \\
\text { arm }(p<0.05) \text { and no significant changes in } \\
\text { the sham arm }\end{array}$ \\
\hline Kim SJ, 2018 [23] & $\begin{array}{l}\text { Significant reduction in the number of freezing episodes from baseline } \\
\text { were seen in the SMA group }(p<0.05) \text { but non-significant change in } \\
\text { MC group } \\
\text { No significant difference in the relative change of FOG-Q post } \\
\text { stimulation between MC and SMA group. }\end{array}$ & $\begin{array}{l}\text { No significant difference score between } \\
\text { MC arm and SMA arm in mean UPDRS total } \\
\text { score } 55.5(17.4), 55.7(11.9) \text { respectively ( } p \\
=0.873 \text { ) } \\
\text { No significant difference score between MC } \\
\text { arm and SMA arm in mean UPDRS III score } \\
28.1 \text { (11.7), } 29.0(8.5) \text { respectively ( } p=0.631 \text { ) }\end{array}$ \\
\hline $\begin{array}{l}\text { Lee, } 2014 \\
{[19]}\end{array}$ & $\begin{array}{l}\text { FOG-Q scores changed from } 13.2(3.4) \text { to } \\
13.0(3.3) \text { in the M1-LL, from } 13.0(3.2) \text { to } 13.0(3.2) \text { in the SMA, from } \\
13.1(3.1) \text { to } 12.9(3.2) \text { in the DLPFC, and from } 13.1(3.2) \text { to } 13.0(3.3) \\
\text { in sham stimulation, respectively; with no significance of any between } \\
\text { pre and post stimulation. }\end{array}$ & $\begin{array}{l}\text { Mean average time for TUG test decreased } \\
\text { when stimulating M1-LL from } 52.7(13.0) \mathrm{sec} \\
\text { to } 38.0(5.8) \mathrm{sec} \text { DLPFC (from } 47.3(9.7 \mathrm{sec}) \\
\text { to } 42.0(8.6) \mathrm{sec} \text { and SMA from } 38.9(7.9) \mathrm{sec} \\
\text { to } 37.0(6.1) \mathrm{sec} \text { with } P=0.003, P=0.046, \\
\text { and } P>0.05 \text { respectively. } \\
\text { Mean UPDRS-III scores were significantly } \\
\text { decreased after both M1-LL from } 22.0(2.5) \\
\text { to } 20.7 \text { ( } 2.5) \text { and DLPFC from } 22.5(2.6) \text { to } \\
21.5(2.5) \text { stimulation }(P=0.002 \text { and } P= \\
0.005 \text { respectively). After SMA and sham } \\
\text { stimulation non-significant change }(P>0.05) \text {. } \\
\text { PD sub-type analysis, only in IPD, there was a } \\
\text { significant improvement in TUG after M1-LL } \\
\text { stimulation only ( } P=0.046) \text {. } \\
\text {, }\end{array}$ \\
\hline
\end{tabular}


Citation: Ibrahim W (2021) Effectiveness of Repetitive Transcranial Magnetic Stimulation on Freezing of Gait in Parkinson's Disease Patients: A Systematic Review of the Literature. J Neurodegener Disord 4(1):101-112

\begin{tabular}{|c|c|c|}
\hline $\begin{array}{l}\text { Rektorova, } 2007 \\
{[21]}\end{array}$ & $\begin{array}{l}\text { FOG-Q scores changed from } 20.5(10 ; 22) \text { to } \\
18.0(12 ; 24) \text { in the M1-LL, and from } 14.0(12 ; 18) \text { to } 17.0(12 ; 18) \text { in the } \\
\text { DLPFC stimulation, respectively; with no significance of any between } \\
\text { pre and post stimulation* } \\
\text { FOG episodes frequency changed from } 10.0(7 ; 14) \text { to } 14.0(1 ; 14) \text { in the } \\
\text { M1-LL, and from } 15.5(11 ; 16) \text { to } 15.0(15 ; 20) \text { in the DLPFC stimulation, } \\
\text { respectively; with no significance of any between pre and post- } \\
\text { stimulation* }\end{array}$ & $\begin{array}{l}\text { UPDRS III changed from } 23.0(9 ; 40) \text { to } 21.0 \\
(9 ; 40) \text { in the M1-LL, and from } 26.0(13 ; 40) \\
\text { to } 22.0(7 ; 40) \text { in the DLPFC stimulation, } \\
\text { respectively; with no significance of any } \\
\text { between pre and post stimulation* }\end{array}$ \\
\hline $\begin{array}{l}\text { Thomassen and } \\
\text { Arns, } 2010[20]\end{array}$ & $\begin{array}{l}\text { FOG-Q scores changed non-significantly from } 6.4(5.5) \text { pre-stimulation } \\
\text { to } 6.7(6.4) \text { post-stimulation }(P=0.795) \text {. }\end{array}$ & $\begin{array}{l}\text { Significant improvement in mean UPDRS total } \\
\text { score } 38.3(15.8) \text { before to } 33.8(17.2) \text { after } \\
\text { stimulation }(P=0.001) \text {. }\end{array}$ \\
\hline
\end{tabular}

*numbers are expressed as median estimates.

The dominant hemisphere was stimulated in six studies (one study stimulated contralateral to more affected side); the other two studies reported stimulation over both hemispheres. neuro-navigational approach was not implemented in any of the included studies in our analysis.

Adverse effects (AE) from rTMS were reported in six studies. The $A E$ reported is mainly headache as most common AE reported $(n=5)$, discomfort during the stimulation $(n=$ $4)$, forehead pain $(n=1)$, and nausea $(n=1)$; although all of them are mild and self-limiting, it led to some patients drop out. Four studies reported a total of 7 drop out patients due to different reasons including acute aggravation of previous heart disease -that was unrelated to the study- $(n=1)$, unbearable forehead pain $(n=1)$, unexpected elbow and shoulder movement $(n=1)$, and transient headache and nausea ( $n$ $=1$ ), while Rektorova and colleague [21] study reported drop out not related to $A E$ which was consent withdrawal $(n=2)$.

All trials implemented FOG-Q being assessment tool for gait as primary and/or secondary outcome, with three studies [21-23] also used frequency of FOG episodes to measure the improvement. TUG test was used as assessment tool for FOG in two studies $[18,19]$.

Assessments of motor functions by MDS-UPDRS were implemented in all studies as secondary outcome. Among UPDRS scale, UPDRS III was used solely in five studies, while UPDRS total score were used in just two studies, and only study by Kim S.J and colleague [23] implemented both UPDRS scores (part III and total score). Other assessment tools were used less frequently as secondary outcome among included studies such as PD-QoL questionnaire (PDQ-39), geriatric depression scale (GDS), and Hoehn and Yahr (H\&Y) stage but was not included in our analysis.

The FoG-Q score showed significant improvement in only three studies, while majority of studies $(n=5)$ showed no statistically significant changes. Meanwhile the UPDRS motor part and/or total score showed significant improvement in majority of studies $(n=5)$, and only three studies reporting no improvement; with two of them showed no improvement in nearly all of primary and/or secondary outcomes measured. The TUG test reported in three studies with improvement in $2 / 3$ of them.

Most of studies $(n=5)$ failed to implement or report fol- low up assessment, and even the remaining three studies reported reassessment after short period of post-stimulation (12 days), with only El-tamawy and colleague [22] study implemented a relatively long follow up period of 3 months.

Finally, there are few ongoing clinical trials (registered on clinicaltrials.gov) for rTMS in treatment of FOG episodes in PD patients. First trial is entitled "rTMS for the Treatment of Freezing of Gait in Parkinson's Disease" [24] a double blind comparative study examining the effectiveness of the rTMS treatment on FOG in 30 PD patients; with estimated Study Completion Date in June, 2019. The second trial is entitled "Repetitive Transcranial Magnetic Stimulation for Freezing of Gait" [25] a Randomized Parallel Assignment of twelve PD patients with FOG to receive rTMS either to MC or SMA (active comparator group); with study Completion Date was July, 2018.

\section{Discussion}

To our knowledge, this is the first systematic review that discusses the effect of rTMS in treatment of FOG in PD patients.

The aim of rTMS treatment in PD is to improve motor symptoms such as bradykinesia, FOG and dyskinesia [13]; however, non-motor symptoms were focus of interest in some trials involving rTMS for depression [26], cognition [27] and speech [28] symptoms in PD patients. Overall, there is a recent growing interest in study the effect of rTMS on PD (motor and non-motor symptoms), other neurodegenerative disorders, and neuropsychiatric diseases [13].

The rTMS studies in general implement different scales or scores for assessment of response to stimulation. The scales include gait assessment (subjective and objective tests) such as FoG-Q, frequency of FOG episodes, and TUG test; scales for motor function assessment such as UPDRS (part III and/or total score) and although UPDRS II contains a single question about FOG it was never used in any study; and other scales for severity/grading of PD such as $H \& Y$, scales for quality of life such as PDQ-39, or scales for comorbidities such as GDS. This great variability in assessment tools represents a great challenge for researchers. A review article by Snijders and colleagues [29] summarizes the difficulties to elicit FOG and available assessment tools with pros and cons of each, and perhaps the most reliable, objective test is quantitative gait 
Citation: Ibrahim W (2021) Effectiveness of Repetitive Transcranial Magnetic Stimulation on Freezing of Gait in Parkinson's Disease Patients: A Systematic Review of the Literature. J Neurodegener Disord 4(1):101-112

analysis which was never implemented in any rTMS trial. This can partially explain the contradictory results between studies were for example some studies showed significant improvement in FoG-Q and/or UPDRS scores or even no significant improvement in neither, other studies showed improvement in different implemented scales as outcome measures.

In regards of brain stimulation region, different sites were stimulated in our included studies and in previous literature. The DLPFC region was stimulated in two trials $[19,21]$ with no significant benefits on FOG; this is expected since previous trials found that stimulating DLFC region has beneficial effect on executive function [30] or depressive symptoms in patients with major depressive disorder [31] rather than on FOG. Dagan and colleague study [32] were the sole study to stimulate the mPFC and no significant improvement on FoG-Q score, with significant improvement in UPDRS III scores; no previous trials for effect of mPFC stimulation on FOG, and only previous literature $[33,34]$ suggest a role in FOG pathophysiology. The dual-mode non-invasive brain stimulation (NIBS) involves simultaneous rTMS and tDCS stimulation had shown greater degree of improvement in terms of FOG-Q outcome compared to rTMS alone in previous studies [35,36], possibly due to their synergistic effect, and whether it should be implemented as superior technique to rTMS alone needs further studies. Another approach never implemented in the included studies is the neuro-navigational approach using MRI scan, while previous studies $[37,38]$ showed enhanced responses to rTMS treatment in setting of the use of neuro-navigational methods to target a specific site.

The different rTMS protocol among studies represented a major challenge and probably the main cause of variability in improvement rate to stimulation. Elahi and colleague [39] review concluded that high-frequency rTMS trials showed significant improvement, meanwhile low frequency trials showed variable results; but this was not the case in our review since all studies used frequency of at least $1 \mathrm{~Hz}$ or more, also frequency was not just the sole factor in influencing the variability in results, this probable due to other cofounding factors in rTMS techniques such as the intensity and duration which affects the response. The sessions number ranged from single session to 16 session with application over variable duration, however in our analysis, the three trials [20,22,32] implemented 10 sessions or more all showed significant improvement in primary and/or secondary outcomes. In final, Elahi and colleague [39], and Fregni and colleagues [14] reviews concluded that different rTMS protocols is an important limitation.

Most of included studies reported the AE from stimulation, and mostly were mild and transient with self-limiting course. The most striking about $A E$ reporting is that Dang and colleague [32] study reported AE in two patients causing drop out from the study, and El-Tamawy and colleague [22] study reported highest number of patient developing AE ( $3 / 8$ patients in real rTMS arm) were both studies used highest number of rTMS session of 16 , and 12 sessions respectively. Previous studies [40] concluded that following appropriate guideline for rTMS stimulation, the results are safe. Meanwhile, increasing the stimulation frequency is associated with more AE including seizures [11]. The international federation of clinical neurophysiology endorsed a number of precautions and recommendations for safe use of rTMS to minimize $A E$ and maximize benefits $[10,11]$.

In sub-group analysis of PD subtypes, only two studies enrolled PPS and VP patients. Lee and colleagues [19] included IPD, VP, and PPS and performed sub-group analysis, and results were significant improvement in IPD and VP groups but not PPS. The other study by Chang and colleagues [18] enrolled only PPS patients without comparative analysis to IPD patients, and results showed significant improvement in both FoG-Q score and UPDRS III compared to sham stimulation. FOG is common in PPS with over $50 \%$ frequency [41] and although it is usually associated with postural instability and falls, the relatively good response in our included studies may be due to the fact that FOG can occur early in PPS [6]. Unfortunately, no enough trials for the effect of rTMS on FOG in literature with sub-group analysis of IPD and VP/PPS patients.

Another important point to be discussed is the long-term clinical relevancy and benefits from rTMS for FOG. All patients were assessed pre and post stimulation immediately and up to 12 days, with only one study by El-tamawy and colleague [22] reassessed patient after 3 months and reported a evident response during follow-up. Unfortunately, there is no enough data from literature about the long term beneficial effect of rTMS on FOG in PD patients, and a systematic review by Elahi and colleague [39] recommended further trials with appropriate follow-up period to determine the long term effect on PD motor symptoms.

The timing of examining the patients was not discussed in depth in most of studies. El-tamawy and colleague [22] reported patients to be assessed during "On state". Lee and colleagues [19] managed to examine patients at the same time of day while their medications remained unchanged.

Another reason for the contradictory or different results in studies in response to rTMS stimulation in spite of relatively matching population characteristics can be explained by small and limited population size and may be variations in rTMS techniques (number of sessions, frequency, and duration). A meta-analysis by Fregni and colleagues [14] concluded that positive effect from the stimulation was not observed in all trials due to small population size of the negative studies.

\section{Strength and limitation points to the review}

The first strength of this review is that it identifies gaps in our current knowledge about therapeutic effect of rTMS on FOG in PD patients. Second, we collected all available articles from inception till December 2018. Third, this review act as a model for future studies investigating the beneficial therapeutic effect of rTMS specifically on FOG in PD patients.

Research in this topic has important limitations that need to be discussed. Firstly, studies vary greatly in samples, methodologies and outcomes measured. Secondly, the FOG assessment tool(s) used varies between studies, with different other tools used as measures of secondary outcome. Third, variability in rTMS protocols including frequency, latency, du- 
Citation: Ibrahim W (2021) Effectiveness of Repetitive Transcranial Magnetic Stimulation on Freezing of Gait in Parkinson's Disease Patients: A Systematic Review of the Literature. J Neurodegener Disord 4(1):101-112

ration and number of sessions, and site of application among studies. Fourth, some studies failed to report duration or severity of PD, medications used, and/or timing of examination.

Furthermore, the difficulties encountered in the current systematic review is that articles reviewed included single arm/non-controlled or non-randomized/pseudorandomized controlled trials, and all studies including RCT enrolled limited numbers of participants.

The result of this systematic review opens up an avenue for the exploration of rTMS effect on FOG among PD patients. To improve the result, further RCT trials enrolling larger number of participants with long follow up period is needed to determine the adequate frequency, duration, number of sessions, and suitable targeted brain region for rTMS in PD patients with FOG.

\section{Conclusion}

Our purpose was to assess the potential beneficial therapeutic effect of rTMS on FOG in PD patients; and due to heterogeneity and low sample size of available studies, and lack of high-quality evidence; only a descriptive analysis of published studies was performed.

From the descriptive analyses, and from the available data of relatively small sample sized studies, it can be concluded that in spite of the above limitations, rTMS had potential beneficial effect in improving the FOG in PD patients with mild $A E$, depending on the used protocol, but we are unable answer whether or not the effect will be sustained for long term. However, our findings encourage further in-depth study of potential clinical value of rTMS on FOG in PD patients.

\section{Disclosure Statement}

No funding was received by the author to carry out the present Systematic review.

The Author has no conflict of interest to declare.

\section{Acknowledgements}

That author expresses his sincere gratitude to Nahla A. Merghany for helping to retrieve all the relevant articles for this review.

\section{References}

1. Zhang L, Canning SD, Wang $X$ (2016) Freezing of gait in parkinsonism and its potential drug treatment. Curr Neuropharmacol 14: 302-306.

2. Snijders AH, Takakusaki K, Debu B, et al. (2016) Physiology of freezing of gait. Ann Neurol 80: 644-659.

3. Okuma Y (2014) Practical approach to freezing of gait in Parkinson's disease. Pract Neurol 14: 222-230.

4. Amboni M, Stocchi F, Abbruzzese G, et al. (2015) Prevalence and associated features of self-reported freezing of gait in Parkinson disease: The DEEP FOG study. Parkinsonism Relat Disord 21: 644-649.

5. Giladi N, Nieuwboer A (2008) Understanding and treating freezing of gait in parkinsonism, proposed working definition, and setting the stage. Mov Disord 23: 423-425.
6. Bloem BR, Hausdorff JM, Visser JE, et al. (2004) Falls and freezing of gait in parkinson's disease : A review of two interconnected, episodic phenomena. Mov Disord 19: 871-884.

7. Cucca A, Biagioni MC, Fleisher JE, et al. (2016) Freezing of gait in Parkinson's disease: From pathophysiology to emerging therapies. Neurodegener Dis Manag 6: 431-446.

8. Fasano A, Herman T, Tessitore A, et al. (2015) Neuroimaging of Freezing of Gait. J Parkinsons Dis 5: 241-254.

9. Mitoma H, Okuma Y (2013) Accelerometry-based long-term monitoring of movement disorders: From diurnal gait behavior to nocturnal bed mobility. J Mech Med Biol 13: 1-22.

10. Wassermann EM (1998) Risk and safety of repetitive transcranial magnetic stimulation : Report and suggested guidelines from the International Workshop on the Safety of Repetitive Transcranial Magnetic Stimulation, June 5-7, 1996. Electroencephalogr Clin Neurophysiol 108: 1-16.

11. Martin PI, Naeser MA, Theoret $\mathrm{H}$, et al. (2004) Transcranial magnetic stimulation as a complementary treatment for aphasia. Semin Speech Lang 25: 181-191.

12. Kim MS, Chang WH, Cho JW, et al. (2015) Efficacy of cumulative high-frequency rTMS on freezing of gait in Parkinson's disease. Restor Neurol Neurosci 33: 521-530.

13. Pallanti S, Marras A (2015) Transcranial magnetic stimulation treatment for motor symptoms in parkinson's disease: A review of two decades of studies Alzheimer's Disease \& Parkinsonism 5: 2.

14. F Fregni, Simon DK, Wu A, et al. (2005) Non-invasive brain stimulation for Parkinson's disease: a systematic review and meta-analysis of the literature. J Neurol Neurosurg Psychiatry 76: 1614-1623.

15. Moher D, Liberati A, Tetzlaff J, et al. (2009) Preferred reporting items for systematic reviews and meta-analyses: the PRISMA statement. Ann Intern Med 151: 264-269.

16. Higgins JPT, Green S (2011) Cochrane handbook for systematic reviews of interventions. The Cochrane Collaboration.Version 5.1.0.

17. Hozo SP, Djulbegovic B, Hozo I (2005) Estimating the mean and variance from the median, range, and the size of a sample. BMC Med Res Methodol 5: 1-10.

18. Chang WH, Kim MS, Cho JW, et al. (2016) Effect of cumulative repetitive transcranial magnetic stimulation on freezing of gait in patients with atypical parkinsonism: A pilot study. J Rehabil Med 48: 824-828.

19. Lee SY, Kim M, Chang WH, et al. (2014) Effects of repetitive transcranial magnetic stimulation on freezing of gait in patients with Parkinsonism. Restor Neurol Neurosci 32: 743-753.

20. Thomassen F, Arns M (2010) Are the Effects of rTMS in Parkinson's Disease Clinically Relevant? J Neurother 14: 96-101.

21. Rektorova I, Sedlackova S, Telecka S, et al. (2007) Repetitive transcranial stimulation for freezing of gait in parkinson's disease. Mov Disord 22: 1518-1519.

22. El-tamawy MS, Shehata HS, Shalaby NM, et al. (2013) Can repetitive transcranial magnetic stimulation help on- freezers with parkinson's disease ? Egypt J Neurol Psychiat Neurosurg 50: 355360.

23. Kim SJ, Paeng SH, Kang SY (2018) Stimulation in supplementary motor area versus motor cortex for freezing of gait in parkinson's disease. J Clin Neurol 14: 320-326. 
Citation: Ibrahim W (2021) Effectiveness of Repetitive Transcranial Magnetic Stimulation on Freezing of Gait in Parkinson's Disease Patients: A Systematic Review of the Literature. J Neurodegener Disord 4(1):101-112

24. rTMS for the Treatment of freezing of gait in parkinson's disease. ClinicalTrials.gov. Identifier: NCT03219892.

25. Repetitive transcranial magnetic stimulation for freezing of gait. ClinicalTrials.gov. Identifier: NCT01853150.

26. Epstein CM, Evatt ML, Funk A, et al. (2007) An open study of repetitive transcranial magnetic stimulation in treatment-resistant depression with parkinson's disease. Clin Neurophysiol 118 : 2189-2194.

27. Furukawa T, Izumi S, Toyokura M, et al. (2009) Effects of low-frequency repetitive transcranial magnetic stimulation in Parkinson's disease. Tokai J Exp Clin Med 34: 63-71.

28. Ae D, Er B, Coracini K, et al. (2006) Effects of repetitive transcranial magnetic stimulation on voice and speech in Parkinson's disease. Acta Neurol Scand 113: 92-99.

29. Snijders AH, Nijkrake MJ, Bakker M, et al. (2008) Clinimetrics of freezing of gait. Mov Disord 23: 468-474.

30. Doruk D, Gray Z, Bravo GL, et al. (2014) Effects of tDCS on executive function in Parkinson's disease. Neurosci Lett 582: 27-31.

31. Boggio PS, Rigonatti SP, Ribeiro RB, et al. (2008) A randomized double-blind clinical trial on the efficacy of cortical direct current stimulation for the treatment of major depression. Int J Neuropsychopharmacol 11: 249-254.

32. Dagan M, Herman T, Mirelman A, et al. (2017) The role of the prefrontal cortex in freezing of gait in Parkinson's disease : Insights from a deep repetitive transcranial magnetic stimulation exploratory study. Exp Brain Res 235: 2463-2472.
33. Nemanich ST, Earhart GM (2016) Freezing of gait is associated with increased saccade latency and variability in Parkinson's disease. Clin Neurophysiol 127: 2394-2401.

34. Gilat M, Shine JM, Bolitho SJ, et al. (2013) Variability of stepping during a virtual reality paradigm in parkinson's disease patients with and without freezing of gait. PLoS One 8: 1-6.

35. Chang WH, Kim MS, Park E, et al. (2017) Effect of dual-mode and dual-site non-invasive brain stimulation on freezing of gait in patients with parkinson's disease. Arch Phys Med Rehabil 98: 1283-1290.

36. Park E, Kim Y, Chang WH, et al. (2014) Interhemispheric modulation of dual-mode, noninvasive brain stimulation on motor function. Ann Rehabil Med 38: 297-303.

37. Fitzgerald PB, Hoy K, Mcqueen S, et al. (2009) A randomized trial of rTMS targeted with MRI based neuro-navigation in treatment-resistant depression. Neuropsychopharmacology 34: 1255-1262.

38. Kim W, Hahn SJ, Kim W, et al. (2016) Neuronavigation-guided repetitive transcranial magnetic stimulation for aphasia. $J$ Vis Exp 6: 1-11.

39. Elahi B, Elahi B, Chen R (2009) Effect of transcranial magnetic stimulation on parkinson motor function -systematic review of controlled clinical trials. Mov Disord 24: 357-363.

40. Wassermann EM, Wedegaertner FR, Ziemann U, et al. (1998) Crossed reduction of human motor cortex excitability by $1-\mathrm{Hz}$ transcranial magnetic stimulation. Neurosci Lett 250: 141-144.

41. Factor SA (2008) The clinical spectrum of freezing of gait in atypical parkinsonism. Mov Disord J 23: 431-438.

DOI: $10.36959 / 459 / 604$

Copyright: (c) $2021 \mathrm{Ibrahim}$ W. This is an open-access article distributed under the terms of the Creative Commons Attribution License, which permits unrestricted use, distribution, and reproduction in any medium, provided the original author and source are credited. 